\title{
Team Meetings within Clinical Domains - Exploring the Use of Routines and Technical Support for Communication
}

\author{
Kristina Groth ${ }^{1,2}$, Ann Lantz ${ }^{1}$, Eva-Lotta Sallnäs ${ }^{1}$, \\ Oscar Frykholm ${ }^{1}$, and Anders Green ${ }^{1}$ \\ ${ }^{1}$ HCI, CSC, Royal Institute of Technology, Stockholm, Sweden \\ ${ }^{2}$ CLINTEC, Karolinska Institutet, Stockholm, Sweden \\ \{kicki, alz, evalotta, frykholm, green\} @csc.kth.se
}

\section{Introduction}

Today, it is common that a team of clinicians, from different disciplines, instead of one single doctor, care for a patient. This is especially true when it concerns more complicated diseases in highly specialised health care. Going from one doctor to a team of doctors raises new dimensions/problems/issues when deciding about the diagnosis and how to treat the patient. Instead of one person deciding, based on the information given from others, a group of people need to agree on a decision. How do the participants during such decision meetings argue for their experience and skill? What kind of technologies are available and how do they support the communication in the meeting? Måseide (2006), for example, focuses on how different forms of evidence influence and regulate the judgements and decisions of medical practitioners during such meetings. Groth et al. (2008), for example, focuses on the technology used during such meetings, with a focus on audio, video, and images.

It has been shown that this kind of multi-disciplinary team meetings increase patient safety (Burton et al. 2006) and that multi-disciplinary site-specialist teams improve processes and outcomes of care (Morris et al. 2006). When comparing operations for liver metastases, Burton et al. reported that decisions made at such meetings showed a higher precision when judging the part of the liver that needs to be removed during the operation.

The objective of this workshop is to gather people with an interest in different aspects on the meeting process in clinical domains. The organisers of the workshop are involved in a project working with technical support in the meeting process at a gastro clinic focusing on highly specialised care. The aim of our project is to explore the use of multi-modal technologies in advanced medical processes such as meetings where physicians from different disciplines meet to decide how to treat a patient.

One focus in our project is to find possibilities to provide relevant information about the patient case discussed during the meeting. For example, if an identified tumour behaves in a specific way, then this together with the patient's general status might be of interest to find similar, previous cases. Cross-referencing of patients, treatment and outcome is today more or less only based on individual physicians' experience and remembrance, not formalised or easily available for others. Another 
focus in our project is to see how haptic feedback can be used in a discussion where radiologist and surgeons discuss a patient case (cf. Anderlind 2008). Radiologists are used to look at radiology pictures and to communicate the radiological diagnosis. The information discussed when looking at the radiology pictures could be further enhanced through other modalities, like touch, in order to better understand the implications for treatment.

The focuses of our project may serve as examples of what we find interesting to discuss in this workshop.

\section{Issues and Goals}

The major focus on the workshop is on team meetings in clinical domains, where several clinicians meet and discuss medical issues. Such meetings may take place both within premises and with participants from remote sites, and may include participants from one or several disciplines. The workshop focuses on the following themes, all in relation to the communication process during team meetings: multimodal interfaces, visualisation of information, participants' interaction with information, haptic interfaces, mediated communication, and other technologies that can support the outcome of the meeting.

Apart from the poster generated as an outcome of the conference, other expected outcomes of this workshop are three-folded: to initiate a network within this area of research, to get a broader perspective of the state of the art research within this area, to initiate future collaborations, organise other workshops or research meetings with the aim of producing prototypes, test scenarios and/or publications.

\section{References}

1. Burton, S., Brown, G., Daniels, I.R.: MRI directed multidisciplinary team preoperative treatment strategy: the way to eliminate positive circumferential margins? Br. J. Cancer 94 (2006)

2. Groth, K., Olin, K., Gran, O., Permert, J.: The role of technology in video-mediated consensus meetings Journal of telemedicine and e-health 14(7) (2008)

3. Morris, E., Haward, R.A., Gilthorpe, M.S., Craigs, C., Forman, D.: The impact of the Calman-Hine report on the processes and outcomes of care for Yorkshire's colorectal cancer patients. Br. J. Cancer 95, 979-985 (2006)

4. Måseide, P.: The deep play of medicine: Discursive and collaborative processing of evidence in medical problem solving. Communication \& Medicine 3(1), 43-54 (2006)

5. Anderlind, E., Noz, M.E., Sallnäs, E.-L., Bengt, K., Lind, B.K., Maguire Jr., G.Q.: Will haptic feedback speed up medical imaging? An application to radiation treatment planning. Journal of Acta Oncologica 47(1), 32-37 (2008) 